\title{
ON LINEAR OPERATORS PRESERVING THE SET OF POSITIVE POLYNOMIALS
}

\author{
ALEXANDER GUTERMAN AND BORIS SHAPIRO
}

To Vladimir Igorevich Arnold with admiration

\begin{abstract}
Following the classical approach of Pólya-Schur theory [14] we initiate in this paper the study of linear operators acting on $\mathbb{R}[x]$ and preserving either the set of positive univariate polynomials or similar sets of non-negative and elliptic polynomials.
\end{abstract}

\section{Contents}

1. Introduction and main results

2. Some preliminaries on the considered classes of preservers 4

$\begin{array}{ll}3 . & \text { The case of diagonal transformations }\end{array}$

3.1. Known correct results $\quad 7$

3.2. Known wrong results 9

4. Linear ordinary differential operators of finite order 10

5. Linear ordinary differential operators with constant coefficients 14

References

\section{INTRODUCTION AND MAIN RESULTS}

Let $\mathbb{R}[x]$ denote the ring of univariate polynomials with real coefficients and denote by $\mathbb{R}_{n}[x]$ its linear subspace consisting of all polynomials of degree less than or equal to $n$.

In what follows we will discuss the following five important types of univariate polynomials:

Definition 1.1. A polynomial $p(x) \in \mathbb{R}[x]$ is called

- hyperbolic, if all its roots are real;

- elliptic, if it does not have reals roots;

- positive, if $p(x)>0$ for all $x \in \mathbb{R}$;

- non-negative, if $p(x) \geq 0$ for all $x \in \mathbb{R}$

- a sum of squares, if there is a positive integer $k$ and there are polynomials $p_{1}(x), \ldots, p_{k}(x) \in \mathbb{R}[x]$ such that $p(x)=p_{1}^{2}(x)+\ldots+p_{k}^{2}(x)$.

Note that the term "elliptic" is sometimes used to define other types of polynomials, see, e.g., [9, 12]. The set of non-negative polynomials is classically compared with the set of sums of squares which is a subset of the latter. Moreover, a wellknown result claims that in the univariate case these two classes coincide, see, e.g., $[17$, p. 132].

2000 Mathematics Subject Classification. Primary: 12D15, 15A04; Secondary: 12D10.

Key words and phrases. Positive, non-negative and elliptic polynomials, linear preservers, Pólya-Schur theory. 
Proposition 1.2. A polynomial $p(x) \in \mathbb{R}[x]$ is non-negative if and only if there exist $p_{1}(x), p_{2}(x) \in \mathbb{R}[x]$ such that $p(x)=p_{1}^{2}(x)+p_{2}^{2}(x)$.

Remark 1.3. Note that the situation is quite different for polynomials in several variables. In particular, even in 2 variables not all non-negative polynomials can be represented as sums of squares. One of the simplest examples of this kind is the polynomial $p(x, y)=x^{2} y^{2}\left(x^{2}+y^{2}-3\right)+1$ which is non-negative but can not be represented as the sum of squares, see [10] for details. In general, this topic is related to the Hilbert 17-th problem, see [13].

Definition 1.4. Let $V$ denote either $\mathbb{R}_{n}[x]$ or $\mathbb{R}[x]$. We say that a map $\Phi: V \rightarrow V$ preserves a certain set $M \subset V$ if for any polynomial $p \in M$ its image $\Phi(p)$ belongs to $M$.

In this paper we study linear operators on $\mathbb{R}[x]$ or $\mathbb{R}_{n}[x]$ which preserve one of the classes of polynomials introduced above. Namely, we call a linear operator acting on $\mathbb{R}[x]$ or $\mathbb{R}_{n}[x]$ a hyperbolicity-, ellipticity-, positivity-, non-negativity-preserver if it preserves the sets of hyperbolic, elliptic, positive, non-negative polynomials respectively. The classical case of (linear) hyperbolicity-preservers which are diagonal in the monomial basis of $\mathbb{R}[x]$ was thoroughly studied about a century ago by Pólya and Schur [14].

Following the set-up of [14] we concentrate below on the remaining three classes of preservers. In short, it turns out that there are much fewer such linear operators than those preserving hyperbolicity. More precisely, our two main results are as follows.

Theorem A. Let $U_{Q}: \mathbb{R}[x] \rightarrow \mathbb{R}[x]$ be a linear ordinary differential operator of order $k \geq 1$ with polynomial coefficients $Q=\left(q_{0}(x), q_{1}(x), \ldots, q_{k}(x)\right), q_{i}(x) \in \mathbb{R}[x]$, $i=0, \ldots, k, q_{k}(x) \not \equiv 0$, i.e.,

$$
U_{Q}=q_{0}(x)+q_{1}(x) \frac{d}{d x}+q_{2}(x) \frac{d^{2}}{d x^{2}}+\ldots+q_{k}(x) \frac{d^{k}}{d x^{k}} .
$$

Then for any coefficient sequence $Q$ the operator $U_{Q}$ does not preserve the set of non-negative (resp., positive or elliptic) polynomials of degree $2 k$.

Corollary. There are no linear ordinary differential operators of positive finite order which preserve the set of non-negative (resp., positive or elliptic) polynomials in $\mathbb{R}[x]$.

Remark 1.5. Notice that by contrast with the above situation there are many hyperbolicity-preservers which are finite order linear differential operators with polynomial coefficients. In fact, such examples exist even among operators with constant coefficients, see Remark 1.6.

Remark 1.6. Any linear operator on $\mathbb{R}[x]$ and $\mathbb{C}[x]$ can be represented as a linear ordinary differential operator of, in general, infinite order, i.e., as a formal power series in $\frac{d}{d x}$ with polynomial coefficients. Thus the subclass of finite order linear differential operators, i.e., those belonging to the Weyl algebra $\mathcal{A}_{1}$ is a natural object of study. Note that unlike the case of finite order operators there exist plenty of linear differential operators of infinite order which preserve positivity. Apparently, the simplest example of this kind is

$$
\left(1-\frac{d}{d x}\right)^{-1}=1+\frac{d}{d x}+\frac{d^{2}}{d x^{2}}+\ldots
$$

More generally, the inverse of any finite order differential operator with constant coefficients and positive constant term whose symbol is a hyperbolic polynomial yields an example of such an operator. Another natural class of such operators 
is obtained by taking the convolution (over $\mathbb{R}$ ) with any positive and sufficiently rapidly decreasing kernel or, in fact, with any positive measure on $\mathbb{R}$ having all finite moments. (Recall that the $k$-th moment of a measure $\mu$ is by definition equal to $\int_{-\infty}^{\infty} t^{k} d \mu(t)$.) The latter observation shows that there are more positivity preservers which are infinite order differential operators with constant coefficients than there are inverses of hyperbolicity-preservers with constant coefficients, see Remark 5.4.

In fact, the latter class coincides with the class of all positivity preservers given by infinite order linear differential operators with constant coefficients. Namely, slightly generalizing a one hundred years old result of Remak [16] and Hurwitz [5] (see also Problem 38 in [15, Ch. 7]) one obtains the following statement.

Theorem B. Let $\alpha=\left(\alpha_{0}, \alpha_{1}, \ldots, \alpha_{k}, \ldots\right)$ be an infinite sequence of real numbers where not all $\alpha_{k}$ vanish. Consider the infinite order linear ordinary differential operator

$$
U_{\alpha}=\alpha_{0}+\alpha_{1} \frac{d}{d x}+\alpha_{2} \frac{d^{2}}{d x^{2}}+\ldots+\alpha_{k} \frac{d^{k}}{d x^{k}}+\ldots
$$

with constant coefficients. Then the operator $U_{\alpha}$ preserves positivity if and only if it preserves non-negativity. Either of requirements is valid if and only if one of the following three equivalent conditions holds:

(1) for any positive (resp., non-negative) polynomial $p(x)=a_{k} x^{k}+\ldots+a_{1} x+a_{0}$ one has that

$$
U_{\alpha}(p)(0)=a_{0} \alpha_{0}+a_{1} \alpha_{1}+\ldots+k ! a_{k} \alpha_{k}>0(\text { resp., } \geq 0) ;
$$

(2) quadratic form represented by any leading principle submatrix of the following infinite Hankel matrix

$$
\left(\begin{array}{cccccc}
\alpha_{0} & 1 ! \alpha_{1} & 2 ! \alpha_{2} & \ldots & l ! \alpha_{l} & \ldots \\
1 ! \alpha_{1} & 2 ! \alpha_{2} & 3 ! \alpha_{3} & \ldots & (l+1) ! \alpha_{l+1} & \ldots \\
2 ! \alpha_{2} & 3 ! \alpha_{3} & 4 ! \alpha_{4} & \ldots & (l+2) ! \alpha_{l+2} & \ldots \\
\vdots & \vdots & \vdots & \ddots & \vdots & \vdots \\
l ! \alpha_{l} & (l+1) ! \alpha_{l+1} & (l+2) ! \alpha_{l+2} & \ldots & (2 l) ! \alpha_{2 l} & \ldots \\
\ldots & \ldots & \ldots & \ldots & \ldots & \ldots
\end{array}\right)
$$

is positive semi-definite (which implies that all leading principal minors of the above matrix are non-negative).

(3) there exists a positive measure $\mu_{\alpha}$ supported on $\mathbb{R}$ whose moments satisfy the relation $\int_{-\infty}^{\infty} t^{k} d \mu_{\alpha}(t)=k ! \alpha_{k}, k=0,1,2, \ldots$. The operator $U_{\alpha}$ can be represented in the form

$$
U_{\alpha}(p)(x)=p(x) \star \mu_{\alpha},
$$

where $p(x) \in \mathbb{R}[x]$ is an arbitrary polynomial and $\star$ denotes the following convolution-like integral transform:

$$
U_{\alpha}(p)(x)=\int_{-\infty}^{\infty} p(t) d \mu(t-x) .
$$

Remark 1.7. The only difference between the operation ' $\star$ ' and the standard convolution is the sign of the argument in the second factor. Notice also that any quadratic form in condition (2) can be positive definite as well, i.e. we assume above that the set of all positive semi-definite quadratic forms includes the set of all positive definite forms as a subset.

To illustrate the latter result notice that for the operator (2) above one has $1=\alpha_{0}=\alpha_{1}=\alpha_{2}=\alpha_{3}=\ldots$ and $\Delta_{l}=\Pi_{j=1}^{l}(j !)^{2}, l=0,1, \ldots$ where $\Delta_{l}$ is the 
corresponding $(l+1) \times(l+1)$ principal minor, see [4]. Another good example is the shift operator $e^{\frac{d}{d x}}=1+\frac{d}{d x}+\frac{d^{2}}{2 ! d x^{2}}+\frac{d^{3}}{3 ! d x^{3}}+\ldots$ for which all entries of the above Hankel matrix are equal to 1.

Remark 1.8. The major remaining challenge in this area is to classify all positivitypreservers. We finish our introduction with this question.

Problem 1. Find a complete classification of positivity-preservers. In particular, describe the semigroup of positivity-preservers which are presented by lower triangular matrices in the monomial basis in $\mathbb{R}[x]$, i.e. which do not increase the degree of a polynomial they are applied to. This semigroup includes two important subsemigroups: a) all positivity preserving differential operators with constant coefficients; b) $\lambda$-sequences discussed in $\S 3$. Strangely enough it is by no means obvious that the latter two subsemigroups generate the former semigroup.

Problem 2. Is there an analog of Theorem B in the multivariate case? In other words, is every positivity preserver which is presented by a linear differential operator with constant coefficients given by the convolution with a positive measure?

Acknowledgments. The authors are grateful to Petter Bränden for important references, to Julius Borcea for discussions, and to Claus Scheiderer for the interest in our work. The first author is sincerely grateful to the Wenner-Gren Foundation, the Swedish Royal Academy of Sciences and the Mittag-Leffler Institute for supporting his visit to Stockholm in Spring 2007 when a substantial part of this project was carried out. The work of the first author was also partially supported by the grants MK-2718.2007.1 and RFBR 08-01-00693a.

\section{Some PRELIMINARIES ON THE CONSIDERED CLASSES OF PRESERVERS}

Below we discuss the relationships between the classes of ellipticity-, positivity-, and non-negativity-preservers. As we mentioned in the introduction the set of all univariate non-negative polynomials coincides with the set of sums of squares and therefore linear preservers of the latter set do not require separate consideration. On the other hand, it is obvious that the sets of elliptic, positive, and non-negative polynomials are distinct. In this section we answer the question about how different are the corresponding sets of ellipticity-, positivity- and non-negativity-preservers, respectively, see Theorems 2.5 and 2.6 below.

We start with the following lemma showing that the assumption that a linear operator $\Phi$ is a non-negativity-preserver is quite strong.

Lemma 2.1. Let $\Phi: \mathbb{R}[x] \rightarrow \mathbb{R}[x]$ be a linear operator preserving the set of nonnegative polynomials. If $\Phi(1) \equiv 0$ then $\Phi \equiv 0$.

Proof. Assume that $\Phi(1) \equiv 0$. First we show that for any polynomial $p(x)=$ $a_{n} x^{n}+\ldots$ of even degree $n=2 m$ one has that if $a_{n}>0$ then $\Phi(p)$ is non-negative and if $a_{n}<0$ then $\Phi(p)$ is non-positive. Indeed, if $n$ is even and $a_{n}>0$ then $p(x)$ has a global minimum, say $M$. Thus $p(x)+|M| \geq 0$ for all $x \in \mathbb{R}$. Therefore, $\Phi(p+|M|)(x) \geq 0$ for all $x \in \mathbb{R}$. However, by linearity and the assumption $\Phi(1) \equiv 0$ we get that

$$
\Phi(p)(x)=\Phi(p)(x)+|M| \cdot 0=\Phi(p)(x)+|M| \Phi(1)(x)=\Phi(p+|M|)(x) \geq 0
$$

for all $x \in \mathbb{R}$. For $a_{n}<0$ the result follows by linearity.

Now let us show that $\Phi(1) \equiv 0$ implies that $\Phi \equiv 0$. Assume that $\Phi \not \equiv 0$ and let $q(x)$ be a polynomial such that $\Phi(q)(x)=a_{n} x^{n}+\ldots+a_{i} x^{i}$ with the smallest possible non-negative value of $i$ such that $a_{i} \neq 0$. Let $p(x)$ be a monic real polynomial of even degree satisfying the condition $\operatorname{deg}(q(x))<\operatorname{deg}(p(x))$. Thus $p(x)+\mu q(x)$ is monic 
for any $\mu \in \mathbb{R}$. The above argument shows that the polynomial $\Phi(p+\mu q)$ is nonnegative for all $\mu \in \mathbb{R}$. Notice that our choice of $q(x)$ implies that the polynomial $\Phi(p)$ has vanishing coefficients at the degrees $0, \ldots, i-1$. Hence $\Phi(p)(x)=b_{l} x^{l}+$ $\ldots+b_{i} x^{i}$ for some positive integer $l$ and some coefficients $b_{l}, \ldots, b_{i} \in \mathbb{R}$. Then for any given $\mu$ there exists $g_{\mu}(x) \in \mathbb{R}[x]$ such that $\Phi(p)(x)+\mu \Phi(q)(x)=x^{i} g_{\mu}(x)$. Obviously, the constant term of $g_{\mu}(x)$ equals $b_{i}+\mu a_{i}$. Since $a_{i} \neq 0$ there exists $\mu_{0} \in \mathbb{R}$ such that $b_{i}+\mu_{0} a_{i}=g_{\mu_{0}}(0)<0$ and by continuity it follows that there exists a neighborhood $N(0)$ of the origin such that $g_{\mu_{0}}(x)<0$ for all $x \in N(0)$. Therefore, there exists $0 \neq x_{0} \in N(0)$ such that $x_{0}^{i}>0$, hence $x_{0}^{i} g_{\mu_{0}}\left(x_{0}\right)<0$. This contradicts the assumption that $\Phi$ is a non-negativity-preserver. Thus $\Phi(q)(x)$ has a vanishing term of degree $i$, which contradicts the choice of $q(x)$. We deduce that $\Phi(q) \equiv 0$ for all $q(x) \in \mathbb{R}[x]$.

Remark 2.2. Notice that Lemma 2.1 is false if we consider a non-negativity preserver in finite degree, i.e. $\Phi: \mathbb{R}_{n}[x] \rightarrow \mathbb{R}_{n}[x]$. A simple example of this sort is obtained by requiring $\Phi(1)=\Phi(x)=\ldots .=\Phi\left(x^{n-1}\right) \equiv 0$ and $\Phi\left(x^{n}\right)=x^{n}$ where $n$ is an even integer.

Theorem 2.3. Let $\Phi: \mathbb{R}[x] \rightarrow \mathbb{R}[x]$ be a linear operator. Then the following conditions are equivalent:

(1) $\Phi$ preserves the set of elliptic polynomials;

(2) either $\Phi$ or $-\Phi$ preserves the set of positive polynomials.

Also each of these conditions implies that

(3) either $\Phi$ or $-\Phi$ preserves the set of non-negative polynomials.

Proof. Note that the identically zero operator satisfies neither condition (1) nor condition (2). Therefore, we will assume that $\Phi \not \equiv 0$.

$(1) \Rightarrow(2)$. Assume that $\Phi$ preserves the set of elliptic polynomials and that neither $\Phi$ nor $-\Phi$ preserves positivity. In other words, since $\Phi$ is an ellipticitypreserver this means that there exist positive polynomials $p(x), q(x) \in \mathbb{R}[x]$ such that $\Phi(p)(x)>0$ and $\Phi(q)(x)<0$ for all $x \in \mathbb{R}$. Note that no elliptic polynomials can be annihilated by $\Phi$ since 0 is not an elliptic polynomial. We consider the following two subcases:

A. There exist two positive polynomials $p(x), q(x)$ as above such that $\operatorname{deg} \Phi(p) \neq$ $\operatorname{deg} \Phi(q)$. Wlog we can assume that $\operatorname{deg} \Phi(p)>\operatorname{deg} \Phi(q)$. Since $\Phi(p)$ is a positive polynomial it has even degree and positive leading coefficient. Thus for any $\mu \in \mathbb{R}$ the polynomial $\Phi(p)+\mu \Phi(q)$ has the same properties, i.e., is of even degree and has positive leading coefficient. Hence there exists $x_{0}(\mu) \in \mathbb{R}$ such that $\Phi(p)(x)+$ $\mu \Phi(q)(x)>0$ for all $x$ with $|x|>x_{0}(\mu)$.

Now set $y_{0}:=\Phi(p)(0)$ and $z_{0}:=\Phi(q)(0)$. Obviously, $y_{0}>0$ and $z_{0}<0$ since $\Phi(p)$ is positive and $\Phi(q)$ is negative. Let $\mu_{0}=\frac{2 y_{0}}{-z_{0}}>0$. Then $p(x)+\mu_{0} q(x)$ is positive since it is the sum of two positive polynomials. At the same time for its image we have that $\Phi\left(p+\mu_{0} q\right)(x)>0$ for $x>x_{0}\left(\mu_{0}\right)$. However, at the origin one has

$$
\Phi\left(p+\mu_{0} q\right)(0)=y_{0}+\frac{2 y_{0}}{-z_{0}} z_{0}=y_{0}-2 y_{0}=-y_{0}<0,
$$

so by continuity $\Phi\left(p+\mu_{0} q\right)(x)$ must have at least one real zero, which is a contradiction.

B. It remains to consider the case when the images of all positive polynomials have the same degree, say $m$. Let $\Phi(p)(x)=a_{m} x^{m}+\ldots, \Phi(q)(x)=b_{m} x^{m}+\ldots$ Since $\Phi(p)(x)>0$ it follows that $a_{m}>0$, and since $\Phi(q)(x)<0$ one has $b_{m}<0$. Thus the polynomial $-b_{m} p(x)+a_{m} q(x)$ is positive. However, its image is of the degree less than $m$, which is a contradiction. 
$(2) \Rightarrow(1)$. If $\Phi$ is a positivity-preserver then by linearity $\Phi$ is also a negativitypreserver, and thus $\Phi$ preserves the set of elliptic polynomials as well.

$(2) \Rightarrow(3)$. Assume that $\Phi$ preserves positivity. Take $p(x) \in \mathbb{R}[x], p(x) \geq 0$. Then for any $\varepsilon>0, p(x)+\varepsilon>0$. Thus $\Phi(p)(x)+\varepsilon \Phi(1)(x)=\Phi(p+\varepsilon)(x)>0$. Taking the limit when $\varepsilon \rightarrow 0$ we get that $\Phi(p) \geq 0$.

The following example shows that, in general, (3) does not imply (1) and (2).

Example 2.4. Let $\Phi: \mathbb{R}[x] \rightarrow \mathbb{R}[x]$ be defined as follows: $\Phi(1)=x^{2}, \Phi\left(x^{i}\right)=0$ for all $i>0$. Obviously, $\Phi$ preserves the set of non-negative polynomials but does not preserve the set of positive polynomials since 1 is mapped to $x^{2}$ which is only non-negative.

We are now going to show that in fact this example is in some sense the only possibility, i.e., it essentially describes the whole distinction between positivity- and non-negativity-preservers.

Theorem 2.5. Let $\Phi: \mathbb{R}[x] \rightarrow \mathbb{R}[x]$ be a non-negativity-preserver. Then either $\Phi$ is a positivity-preserver (and therefore an ellipticity-preserver as well) or $\Phi(1)$ is a polynomial which is only non-negative but not positive. Moreover, in the latter case for any positive polynomial $p(x) \in \mathbb{R}[x]$ the zero locus of $\Phi(p)$ is a subset of the zero locus of $\Phi(1)$.

Proof. Assume that $\Phi \not \equiv 0$ is a non-negativity-preserver. Then $\Phi$ sends positive polynomials to non-negative ones. Let us assume that $p(x) \in \mathbb{R}[x]$ is positive but its image $\Phi(p)(x)$ has real zeros. Since $p(x)$ is positive there exists $\varepsilon>0$ such that $p(x)-\varepsilon>0$ for all $x$. Thus for its image we have

$$
\Phi(p-\varepsilon)(x)=\Phi(p)(x)-\varepsilon \Phi(1)(x) \geq 0 .
$$

Set $g(x):=\Phi(p)(x), f(x):=\Phi(p-\varepsilon)(x)$, and $h(x):=\Phi(1)(x)$. Since all three polynomials are non-negative and $g(x)=\varepsilon h(x)+f(x)$, it follows that for any $x_{0}$ such that $g\left(x_{0}\right)=0$ one has that $f\left(x_{0}\right)=h\left(x_{0}\right)=0$. Since $h(x)$ is a polynomial then either $h(x) \equiv 0$, or $h(x)$ has a finite number of zeros. However, the first possibility is ruled out by Lemma 2.1 , since $h(x)=\Phi(1)$. The second possibility implies that all positive polynomials whose images are non-negative but not positive have altogether only a finite number of zeros belonging to the zero locus of $\Phi(1)(x)$.

Corollary 2.6. Let $\Phi: \mathbb{R}[x] \rightarrow \mathbb{R}[x]$ be a linear operator such that the polynomial $\Phi(1)>0$. Then the conditions (1), (2) and (3) of Theorem 2.3 are equivalent.

In exactly the same way we can show the following.

Theorem 2.7. Let $\Phi: \mathbb{R}_{n}[x] \rightarrow \mathbb{R}_{n}[x]$ be a linear operator with $\Phi(1)>0$. Then the following conditions are equivalent:

(1) $\Phi$ preserves the set of elliptic polynomials of degree $\leq n$;

(2) either $\Phi$ or $-\Phi$ preserves the set of positive polynomials of degree $\leq n$;

(3) either $\Phi$ or $-\Phi$ preserves the set of non-negative polynomials of degree $\leq n$.

Remark 2.8. Corollary 2.6 and Theorem 2.7 will allow us to reduce the investigation of non-negativity-, positivity-, and ellipticity-preservers (both in the finitedimensional and the infinite-dimensional cases) to just one of these three classes of preservers.

For the sake of completeness notice that for non-linear operators the situation is different from the one above as the following simple examples show.

Example 2.9. 1. The bijective map $\Phi_{1}: \mathbb{R}[x] \rightarrow \mathbb{R}[x]$ defined by

$$
\Phi_{1}(p)(x)=p(x)+c
$$


where $c$ is a positive constant, preserves both positivity and non-negativity but does not preserve ellipticity.

2. The bijective map $\Phi_{2}: \mathbb{R}[x] \rightarrow \mathbb{R}[x]$ defined by

$$
\Phi_{2}(p)(x)= \begin{cases}p(x) & \forall p(x) \in \mathbb{R}[x] \backslash\left\{x^{2}+1,-x^{2}-1\right\} \\ -x^{2}-1 & \text { if } p(x)=x^{2}+1 \\ x^{2}+1 & \text { if } p(x)=-x^{2}-1\end{cases}
$$

preserves ellipticity, but does not preserve positivity and non-negativity.

3. The bijective map $\Phi_{3}: \mathbb{R}[x] \rightarrow \mathbb{R}[x]$ defined by

$$
\Phi_{3}(p)(x)= \begin{cases}p(x) & \forall p(x) \in \mathbb{R}[x] \backslash\left\{ \pm x^{2}\right\} \\ -x^{2} & \text { if } p(x)=x^{2} \\ x^{2} & \text { if } p(x)=-x^{2}\end{cases}
$$

preserves ellipticity and positivity, but does not preserve non-negativity.

\section{THE CASE OF DiAgONAL TRANSFORMATIONS}

The aim of this section is twofold. Firstly we want to recall what was previously known about positivity- and non-negativity-preservers in the classical case of linear operators acting diagonally in the standard monomial basis of $\mathbb{R}[x]$ and secondly we want to point out some (known to the specialists in the field, [2], [3]) mistakes in Ch.4 of the important treatise [6]. The main source of correct results is Ch. 3 of $[17]$.

3.1. Known correct results. Let $T_{\infty}: \mathbb{R}[x] \rightarrow \mathbb{R}[x]$ be a linear operator defined by

$$
T\left(x^{i}\right)=\lambda_{i} x^{i} \text { for } i=0,1, \ldots
$$

and, analogously, let $T_{n}: \mathbb{R}_{n}[x] \rightarrow \mathbb{R}_{n}[x]$ be a linear operator defined by

$$
T_{n}\left(x^{i}\right)=\lambda_{i} x^{i} \text { for } i=0,1, \ldots, n .
$$

Denote them by $\left\{\lambda_{i}\right\}_{i=0}^{\infty}$ and $\left\{\lambda_{i}\right\}_{i=0}^{n}$, respectively. We will refer to such operators as diagonal transformations or diagonal sequences. Diagonal transformations preserving the set of positive polynomials are referred to as $\lambda$-sequences in the literature, see [6], p.110, and [2,3,8]. Reserving the symbol $\Phi$ for general linear operators we use in this section the notation $T \in \Lambda$ to emphasize that $T$ is a diagonal transformation preserving positivity.

Remark 3.1. Notice that in the finite-dimensional case we only need to consider transformations acting on $\mathbb{R}_{n}[x]$ for $n$ even since there are no positive polynomials of odd degree and a sequence $\left\{\lambda_{i}\right\}_{i=0}^{2 k+1}$ preserves the set of positive polynomials in $\mathbb{R}_{2 k+1}$ if and only if $\left\{\lambda_{i}\right\}_{i=0}^{2 k}$ preserves the set of positive polynomials in $\mathbb{R}_{2 k}$.

Let us establish some immediate consequences of the fact that a diagonal transformation $T$ is a positivity-preserver.

Lemma 3.2. Assume that a transformation $T_{\alpha}=\left\{\lambda_{i}\right\}_{i=0}^{\alpha}, \alpha \in \mathbb{N} \cup\{\infty\}$, preserves positivity. Then

(1) $\lambda_{i} \geq 0$ for any even $i$;

(2) $\lambda_{i}^{2} \leq \lambda_{0} \lambda_{2 i}$ for any $i$.

Proof. To settle (1) consider the polynomial $p(x)=x^{i}+1$ which is positive if $i$ is even. Thus $T(p)=\lambda_{i} x^{i}+\lambda_{0}$ should be positive as well. Since $\lambda_{0}>0$, the result follows.

To settle (2) consider the polynomial $p(x)=x^{2 i}+a x^{i}+b$ with $a^{2}<4 b$. Then $p(x)$ is positive as well as its image $q(x):=T(p)(x)=\lambda_{2 i} x^{2 i}+a \lambda_{i} x^{i}+b \lambda_{0}$. If $i$ is odd 
then the positivity of $q(x)$ is equivalent to the negativity of its discriminant, i.e., $D_{q}:=a^{2} \lambda_{i}^{2}-4 b \lambda_{0} \lambda_{2 i}<0$, which implies $\lambda_{i}^{2} \leq \lambda_{0} \lambda_{2 i}$ since $a^{2}<4 b$. Finally, if $i$ is even then $q(x)$ is positive iff either $D_{q}<0$ or $D_{q}>0$ and if additionally both roots of the quadratic polynomial $\lambda_{2 i} z^{2}+a \lambda_{i} z+b \lambda_{0}$ are negative. In the first subcase one has $\lambda_{i}^{2} \leq \lambda_{0} \lambda_{2 i}$ as for $i$ odd. In the second subcase we obtain that the positive polynomial $x^{2 i}-a x^{i}+b$ is transformed to the polynomial $\lambda_{2 i} x^{2 i}-a \lambda_{i} x^{i}+b \lambda_{0}$ which has some real roots. To check this notice that the roots of $\lambda_{2 i} z^{2}-a \lambda_{i} z+b \lambda_{0}$ are opposite to that of $\lambda_{2 i} z^{2}+a \lambda_{i} z+b \lambda_{0}$ and are, therefore, positive. Thus extracting their $i$-th root one will get some positive roots as well. This contradiction shows that the inequality $\lambda_{i}^{2} \leq \lambda_{0} \lambda_{2 i}$ is necessary for the positivity-preservation.

We will need the following class of sequences of real numbers, see [17], p. 127. (The terminology does not seem to be very satisfactory but we preserve it to avoid further confusion.)

Definition 3.3. A sequence $\left\{\lambda_{i}\right\}_{i=0}^{\alpha}$ is called positive if for any non-negative polynomial $p(x)=x^{n}+a_{n-1} x^{n-1}+\ldots+a_{1} x+a_{0} \in \mathbb{R}_{\alpha}[x]$ one has that $\lambda_{n}+a_{n-1} \lambda_{n-1}+$ $\ldots+a_{1} \lambda_{1}+a_{0} \lambda_{0} \geq 0$, i.e., $T_{\alpha}(p)(1) \geq 0$.

In the infinite-dimensional case the following characterizations of the set of diagonal positivity-preservers, i.e. $\lambda$-sequences is attributed to Iliev who in his turn refers to [11] p. 453 in the very first lines of $\S 4.3$, [6]. Iliev's statement is slightly wrong and as such was cited in [2], [3, Theorem 1.7], see also $\S 3.2$ below. The correct statement is as follows.

Theorem 3.4. Let $\left\{\lambda_{k}\right\}_{k=0}^{\infty}$ be a sequence of real numbers not all vanishing. Then the following conditions are equivalent:

(1) The transformation $T: \mathbb{R}[x] \rightarrow \mathbb{R}[x]$ generated by the sequence $\left\{\lambda_{k}\right\}_{k=0}^{\infty}$ as in formula (4) preserves the set of non-negative polynomials;

(2) $\left\{\lambda_{k}\right\}_{k=0}^{\infty}$ is a positive sequence;

(3) For any nonnegative integer $k$ the quadratic form given by the Hankel matrix

$$
\left(\lambda_{i+j}\right)=\left(\begin{array}{cccc}
\lambda_{0} & \lambda_{1} & \ldots & \lambda_{k} \\
\lambda_{1} & \lambda_{2} & \ldots & \lambda_{k+1} \\
\vdots & \vdots & \ddots & \vdots \\
\lambda_{k} & \lambda_{k+1} & \ldots & \lambda_{2 k}
\end{array}\right)
$$

is positive semi-definite. (Recall that we assume that any positive definite quadratic form is also positive semi-definite by definition);

(4) There exists a non-decreasing function $\mu(t)$ such that for all $k=0,1,2, \ldots$ one has

$$
\lambda_{k}=\int_{-\infty}^{\infty} t^{k} d \mu(t)
$$

Proof. The equivalences $(2) \Leftrightarrow(3) \Leftrightarrow(4)$ are settled in $[17$, p. 129 , Theorem 10 and p. 133, Theorem 11] independently of condition (1) above. The implication $(1) \Rightarrow(2)$ is evident so we only have to concentrate on the remaining implication $(2) \Rightarrow(1)$. Take a non-negative polynomial $g(x)=a_{l} x^{l}+\ldots+a_{1} x+a_{0}$ and set $q(x):=T(g)(x)=\sum_{i=0}^{l} \lambda_{i} a_{i} x^{i}$. We want to show that $q(x) \geq 0$ for all real $x$. 
Condition (4) implies that $\lambda_{i}=\int_{-\infty}^{\infty} t^{i} d \mu(t)$, where $\mu(t)$ is a monotone nondecreasing function. Hence

$$
q(x)=\sum_{i=0}^{l}\left(\int_{-\infty}^{\infty} t^{i} d \mu(t)\right) x^{i}=\int_{-\infty}^{\infty} \sum_{i=0}^{l} a_{i} t^{i} x^{i} d \mu(t)=\int_{-\infty}^{\infty} g(x t) d \mu(t) \geq 0
$$

since $g(x t) \geq 0$ for all $t$. Notice that the above integrals are convergent for any fixed value of $x$. Thus $q(x) \geq 0$ and the theorem follows.

The set of all positive sequences is naturally divided into two subsets: positive definite sequences and positive semi-definite sequences.

Definition 3.5. A sequence $\left\{\lambda_{i}\right\}_{i=0}^{\infty}$ is called positive definite if for any nonnegative polynomial $p(x)=x^{n}+a_{n-1} x^{n-1}+\ldots+a_{1} x+a_{0} \in \mathbb{R}[x]$ which is not identically zero one has that $\lambda_{n}+a_{n-1} \lambda_{n-1}+\ldots+a_{1} \lambda_{1}+a_{0} \lambda_{0}>0$. Analogously, a sequence $\left\{\lambda_{i}\right\}_{i=0}^{\infty}$ is called positive semi-definite if there exists a non-negative polynomial $p(x)=x^{n}+a_{n-1} x^{n-1}+\ldots+a_{1} x+a_{0} \in \mathbb{R}[x]$ which is not identically zero such that $\lambda_{n}+a_{n-1} \lambda_{n-1}+\ldots+a_{1} \lambda_{1}+a_{0} \lambda_{0}=0$.

A nice characterization of these two subclasses is given in $[17$, p. 134 , Theorem $12 \mathrm{a}]$.

Theorem 3.6. A sequence $\left\{\lambda_{i}\right\}_{i=0}^{\infty}$ is positive definite (resp. semi-definite) if and only if there exists a non-decreasing function $\mu(t)$ with infinitely many points of increase (resp. with finitely many points of increase) such that for all $k=0,1,2, \ldots$ one has

$$
\lambda_{k}=\int_{-\infty}^{\infty} t^{k} d \mu(t)
$$

Remark 3.7. Notice that for a positive definite sequence every quadratic form given in (3) of Theorem 3.4 is positive definite which is equivalent to the positivity of all principal minors of the corresponding Hankel matrix. In the case of positive semi-definite sequence the corresponding minors are non-negative but if one has a Hankel matrix with all non-negative principal minors one can not conclude that the corresponding quadratic form is positive semi-definite, see p. 136 of [17].

Remark 3.8. Notice that by Lemma 2.1 any nontrivial positive sequence $\left\{\lambda_{i}\right\}_{i=0}^{\infty}$ has to have $\lambda_{0}>0$ and then it is also a positivity preserver.

3.2. Known wrong results. L. Iliev claims in the beginning of $\S 4.3$. of [6] that the class of $\lambda$-sequences coincides with the class of positive definite sequences which is immediately disproved by the sequence all entries of which are equal to 1 , i.e. by the identity operator.

To present some further erroneous results from [6] and the corresponding counterexamples we need to introduce the following classes of diagonal transformations.

Definition 3.9. We say that $T_{\alpha}, \alpha \in \mathbb{N} \cup \infty$, or, equivalently, the sequence $\left\{\lambda_{i}\right\}_{i=0}^{\alpha}$, is a hyperbolicity-preserver, if for any hyperbolic $p(x) \in \mathbb{R}_{\alpha}[x]$ its image $T_{\alpha}(p)(x)$ is hyperbolic. We denote this class of transformations by $\mathcal{H}_{\alpha}$ or $\mathcal{H}$ and the class of all $\lambda$-sequences by $\Lambda$.

Clearly, this class is the restriction of the earlier defined class of hyperbolicitypreservers to diagonal transformations.

Theorem 4.6.14 of [6] states that $T \in \Lambda$ if and only if $T^{-1} \in \mathcal{H}$. We will now show that this statement is wrong in both directions. 
Proposition 3.10. There exist

(i) $T \in \Lambda$ such that $T^{-1} \notin \mathcal{H}$;

(ii) $T \in \mathcal{H}$ such that $T^{-1} \notin \Lambda$.

Proof. We present below 3 concrete examples verifying the above claims. To illustrate (i) consider the diagonal transformation $T_{4}: \mathbb{R}_{4}[x] \rightarrow \mathbb{R}_{4}[x]$ defined by the sequence $\left(\lambda_{0}, \lambda_{1}, \lambda_{2}, \lambda_{3}, \lambda_{4}\right)=\left(\frac{1}{29}, \frac{1}{68}, \frac{1}{123}, \frac{1}{200}, \frac{1}{305}\right)$. By (3) of Theorem 3.4 and Remark 3.8 the operator $T_{4}$ preserves positivity. However, one can check that its inverse sends the non-negative polynomial $(x+1)^{4}$ to the polynomial $(x+1)\left(305 x^{3}+495 x^{2}+243 x+29\right)$ possessing two real and two complex roots.

This example shows that in the finite-dimensional case there is a diagonal transformation which preserves positivity, but whose inverse does not preserve hyperbolicity. We can extend this example to the infinite-dimensional case as follows.

By [2, Proposition 3.5] there exists an infinite sequence $\left\{\lambda_{i}\right\}_{i=0}^{\infty} \in \Lambda$ such that the sequence of inverses $\left\{\frac{1}{\lambda_{i}}\right\}_{i=1}^{\infty} \notin \mathcal{H}$. As an explicit example one can take $\lambda_{i}=\frac{1}{i^{3}+5 i^{2}+33 i+29}$.

An example illustrating (ii) is given in [2, p. 520], see also [3, Example 1.8]. Namely, the sequence $\left\{1+i+i^{2}\right\}_{i=0}^{\infty}$ corresponds to a diagonal transformation preserving hyperbolicity. However, the sequence of inverses $\left\{\frac{1}{1+i+i^{2}}\right\}_{i=0}^{\infty}$ leads to a diagonal transformation which is not a positivity-preserver.

Definition 3.11. We say that a diagonal transformation $T_{\alpha}, \alpha \in \mathbb{N} \cup \infty$, defined by the sequence $\left\{\lambda_{i}\right\}_{i=0}^{\alpha}$ is a complex zero decreasing sequence (CZDS for short), if for any polynomial $p(x) \in \mathbb{R}_{\alpha}[x]$ the polynomial $T(p)$ has no more non-real roots (counted with multiplicities) than $p$. We denote the set of all CZDS by $\mathcal{R}$.

Remark 3.12. Obviously, any CZDS preserves hyperbolicity, i.e., $\mathcal{R} \subset \mathcal{H}$. For a while it was believed that $\mathcal{R}=\mathcal{H}$ until Craven and Csordas found a counterexample [2]. Additionally, one can see directly from the definition that the inverse of any positive CZDS is a $\Lambda$-sequence, that is, a diagonal positivity-preserver.

Finally, Theorem 4.6 .13 of [6] states that $T \in \Lambda$ if and only if $T^{-1} \in \mathcal{R}$, which we disprove below.

Proposition 3.13. There exist $T \in \Lambda$ such that $T^{-1} \notin \mathcal{R}$.

Proof. Use the first two counterexamples from the proof of Proposition 3.10.

\section{LiNEAR ORDINARY DIFFERENTIAL OPERATORS OF FINITE ORDER}

Our aim in this section is to prove Theorem A, i.e., to show that there are no positivity-, non-negativity-, and ellipticity-preservers which are linear differential operators of finite positive order. In fact we are going to show that for any linear differential operator $U$ of order $k \geq 1$ there exists an integer $n$ such that $U: \mathbb{R}_{n}[x] \rightarrow$ $\mathbb{R}_{n}[x]$ is not a non-negativity preserver. Moreover, we show that one can always choose $n=2 k$. Since any positivity-preserver is automatically a non-negativitypreserver and any ellipticity-preserver is a positivity-preserver up to a sign change we will get Theorem A in its complete generality from the above statement.

Denote by $S\left[s_{1}, \ldots, s_{k}\right]$ the ring of symmetric polynomials with real coefficients in the variables $s_{1}, \ldots, s_{k}$. Let $\sigma_{l}$ be the $l$-th elementary symmetric function, i.e.,

$$
\sigma_{l}=\sum_{j_{1}<\ldots<j_{l}} s_{j_{1}} \cdots s_{j_{l}} \in S\left[s_{1}, \ldots, s_{k}\right], \quad l=1, \ldots, k .
$$


We will need the following technical fact.

Proposition 4.1. Let $p(x)=\left(x-x_{1}\right)^{2} \cdots\left(x-x_{k}\right)^{2} \in \mathbb{R}\left[x, x_{1}, \ldots, x_{k}\right]$. Consider the following two families of rational functions:

$$
\begin{aligned}
& w_{l}=w_{l}\left(x, x_{1}, \ldots, x_{k}\right)=\frac{p^{(l)}(x)}{p(x)}, \quad l=1, \ldots, k \\
& u_{l}=\sigma_{l}\left(\frac{1}{x-x_{1}}, \ldots, \frac{1}{x-x_{k}}\right), \quad l=1, \ldots, k
\end{aligned}
$$

Then

1. $w_{l} \in S\left[\frac{1}{x-x_{1}}, \ldots, \frac{1}{x-x_{k}}\right], l=1, \ldots, k$.

2. For any $l=1, \ldots, k$ one has that

$$
w_{l}=2 l ! u_{l}+g_{l}\left(u_{1}, \ldots, u_{l-1}\right),
$$

where $g_{l} \in \mathbb{R}\left[y_{1}, \ldots, y_{l-1}\right], l=1,2, \ldots, k$, are certain polynomials (that can be found explicitly but we will not need their explicit form; in particular, $g_{1} \equiv 0$ ).

Proof. Set $p_{1}(x)=\left(x-x_{1}\right) \cdots\left(x-x_{k}\right)$. Then one can immediately check that

$$
\frac{p_{1}^{(i)}(x)}{p_{1}(x)}=i ! u_{i}
$$

for all $i=1, \ldots, k$. Using the Leibniz rule we get

$$
p^{(l)}(x)=\left(p_{1}^{2}(x)\right)^{(l)}=2 p_{1}^{(l)}(x) p_{1}(x)+\sum_{i, j \geq 1, i+j=l} c_{i, j} p_{1}^{(i)}(x) p_{1}^{(j)}(x),
$$

where $c_{i, j} \geq 0$ are certain binomial coefficients. Thus

$w_{l}=\frac{p^{(l)}(x)}{p(x)}=2 \frac{p_{1}^{(l)}(x)}{p_{1}(x)}+\sum_{i, j \geq 1, i+j=l} c_{i, j} \frac{p_{1}^{(i)}(x)}{p_{1}(x)} \cdot \frac{p_{1}^{(j)}(x)}{p_{1}(x)}=2 l ! u_{l}+\sum_{i, j \geq 1, i+j=l} c_{i, j} u_{i} u_{j}$.

The result follows.

We are now ready to prove the first main result of this paper.

Theorem 4.2. Let $U_{Q}: \mathbb{R}[x] \rightarrow \mathbb{R}[x]$ be a linear ordinary differential operator of order $k \geq 1$ of the form (1) with polynomial coefficients $Q=\left(q_{0}(x), q_{1}(x), \ldots, q_{k}(x)\right)$, $q_{i} \in \mathbb{R}[x], q_{k}(x) \not \equiv 0$. Then for any such coefficient sequence $Q$ the operator $U_{Q}$ does not preserve the set of non-negative polynomials of degree $2 k$.

Proof. We assume that $U_{Q} \not \equiv 0$. Since $U_{Q}(1)=q_{0}(x)$ an obvious necessary condition for the operator $U_{Q}$ to preserve non-negativity is that $q_{0}(x)$ itself is a nonnegative polynomial. Moreover, $q_{0}(x)$ does not vanish identically by Lemma 2.1 .

We will now construct a non-negative polynomial

$$
p(x):=p_{x_{1}, \ldots, x_{k}}(x)=\left(x-x_{1}\right)^{2}\left(x-x_{2}\right)^{2} \cdots\left(x-x_{k}\right)^{2} \in \mathbb{R}\left[x, x_{1}, \ldots, x_{k}\right] .
$$

such that its image under the action of $U_{Q}$ attains negative values. For this we define

$R\left(x, x_{1}, \ldots, x_{k}\right)=\frac{U_{Q}(p)(x)}{p(x)}=q_{0}(x)+q_{1}(x) \frac{p^{\prime}(x)}{p(x)}+q_{2}(x) \frac{p^{\prime \prime}(x)}{p(x)}+\ldots+q_{k}(x) \frac{p^{(k)}(x)}{p(x)}$.

Then in the notation of Proposition 4.1 we have that $R\left(x, x_{1}, \ldots, x_{k}\right)=q_{0}(x)+$ $q_{1}(x) w_{1}+\ldots+q_{k}(x) w_{k}$. Let us fix $x_{0} \in \mathbb{R}$ such that $x_{0} \neq 0$ and for any $i=$ $0,1, \ldots, k$ either $q_{i}(x) \equiv 0$ or $q_{i}\left(x_{0}\right) \neq 0$. Set $\alpha_{i}=q_{i}\left(x_{0}\right), i=1, \ldots, k$, and $r\left(x_{1}, \ldots, x_{k}\right)=R\left(x_{0}, x_{1}, \ldots, x_{k}\right)$. Then $r\left(x_{1}, \ldots, x_{k}\right)$ is a linear form in $w_{1}^{\prime}, \ldots, w_{k}^{\prime}$, 
where $w_{i}^{\prime}=w_{i}\left(x_{0}\right)$. Thus there exist $a_{1}, \ldots, a_{k} \in \mathbb{R}$ such that $q_{0}\left(x_{0}\right)+\sum_{i=1}^{k} \alpha_{i} a_{i}<0$.

(Notice that by our choice of $x_{0}$ one has $q_{0}\left(x_{0}\right)>0$.)

Let now $b_{1}, \ldots, b_{k} \in \mathbb{R}$ be defined by

$$
b_{1}=a_{1}, \quad b_{i}=\frac{1}{2 i !}\left(a_{i}-g_{i}\left(b_{1}, \ldots, b_{i-1}\right)\right), \quad i=2, \ldots, k,
$$

where $g_{i}$ are defined in Proposition 4.1. Consider the system of equations

$$
b_{i}=\sigma_{i}\left(\frac{1}{x_{0}-t_{1}}, \ldots, \frac{1}{x_{0}-t_{k}}\right), \quad i=1, \ldots, k,
$$

with unknowns $t_{1}, \ldots, t_{k}$. It follows from the Vièta theorem that the $k$-tuple $\left(t_{1}, \ldots, t_{k}\right)$ solves system (8) if and only if $\frac{1}{x_{0}-t_{1}}, \ldots, \frac{1}{x_{0}-t_{k}}$ are the roots of the equation

$$
z^{k}-b_{1} z^{k-1}+b_{2} z^{k-2}-\ldots \pm b_{k-1} z \mp b_{k}=0 .
$$

Note that since $b_{i} \in \mathbb{R}$ the roots of the latter equation are either real or complex conjugate. Wlog we can always assume that they are ordered so that $z_{1}=$ $\overline{z_{2}}, \ldots, z_{2 i-1}=\overline{z_{2 i}} \in \mathbb{C}, z_{2 i+1}, \ldots, z_{k} \in \mathbb{R}$. Thus the $k$-tuple $\left(t_{1}, \ldots, t_{k}\right)$, where $t_{i}=x_{0}-\frac{1}{z_{i}}, i=1, \ldots, k$, solves system (8). Obviously, $t_{1}=\overline{t_{2}}, \ldots, t_{2 i-1}=\overline{t_{2 i}} \in \mathbb{C}$, $t_{2 i+1}, \ldots, t_{k} \in \mathbb{R}$.

Let us substitute $x=x_{0}, x_{i}=t_{i}, i=1, \ldots, k$, in the functions $w_{l}, l=1, \ldots, k$, defined in Proposition 4.1. By the definition of the $u_{i}$ 's and using the fact that the $t_{i}$ 's solve system (8) we get that $u_{i}\left(x_{0}, t_{1}, \ldots, t_{k}\right)=b_{i}, i=1, \ldots, k$. Thus (7) implies that

$$
w_{i}\left(x_{0}, t_{1}, \ldots, t_{k}\right)=a_{i}, \quad i=1, \ldots, k .
$$

Hence $R\left(x_{0}, t_{1}, \ldots, t_{k}\right)<0$ by the choice of the $a_{i}$ 's. Thus $U_{\alpha}\left(p_{t_{1}, \ldots, t_{k}}\right)\left(x_{0}\right)<0$ since $p_{x_{1}, \ldots, x_{k}}(x) \geq 0$ for all $x \in \mathbb{R}$ and all $x_{i}, i=1, \ldots, k$. This contradiction proves Theorem 4.2.

As we mentioned above Theorem 4.2 together with the results of $\S 2$ imply Theorem A.

We are now going to strengthen Theorem 4.2 and show that wide subclasses of linear ordinary differential operators of finite order $k$ do not preserve non-negativity even in degrees much smaller than $2 k$. In particular, the next statement shows that no linear differential operator of odd order $k$ preserves the set of non-negative polynomials in $\mathbb{R}_{k+1}[x]$.

Proposition 4.3. Let $U_{Q}: \mathbb{R}[x] \rightarrow \mathbb{R}[x]$ be a linear differential operator of odd order $k \geq 1$ of the form (1) with polynomial coefficients $Q=\left(q_{0}(x), q_{1}(x), \ldots, q_{k}(x)\right)$, $q_{i}(x) \in \mathbb{R}[x], i=1, \ldots, k, q_{k}(x) \not \equiv 0$. Then $U_{Q}$ does not preserve the set of nonnegative polynomials of degree smaller than or equal to $k+1$.

Proof. Consider the polynomial $p_{t}(x)=(x-t)^{k+1}$. It is non-negative since $k$ is odd. Note that

$$
r_{Q}(x, t):=\frac{U_{Q}\left(p_{t}\right)(x)}{p_{t}(x)}=q_{0}(x)+(k+1) q_{1}(x) \frac{1}{x-t}+\ldots+(k+1) ! q_{k}(x) \frac{1}{(x-t)^{k}}
$$

and set $u:=\frac{1}{x-t}$. Then the function

$$
g(x, u):=q_{0}(x)+(k+1) q_{1}(x) u+\ldots+(k+1) ! q_{k}(x) u^{k}
$$

is a polynomial in $u$. Fixing $x_{0}$ such that $q_{k}\left(x_{0}\right) \neq 0$ we obtain that $g\left(x_{0}, u\right)$ is a polynomial in $u$ of odd degree. Hence there exists $u_{0}$ such that $g\left(x_{0}, u_{0}\right)<0$. Now for $t_{0}=x_{0}-\frac{1}{u_{0}}$ we get that $r_{Q}\left(x_{0}, t_{0}\right)<0$. Thus $U_{Q}\left(p_{t_{0}}\right)\left(x_{0}\right)<0$ since $p_{t}(x) \geq 0$ for all $(x, t)$. This contradiction finishes the proof. 
The next result shows that there is also a large class of linear differential operators of even order $k$ which does not preserve non-negativity in $\mathbb{R}_{k}[x]$.

Proposition 4.4. Let $U_{Q}: \mathbb{R}[x] \rightarrow \mathbb{R}[x]$ be a linear differential operator of even order $k$ of the form (1) with polynomial coefficients $Q=\left(q_{0}(x), q_{1}(x), \ldots, q_{k}(x)\right)$, $q_{i}(x) \in \mathbb{R}[x], i=1, \ldots, k, q_{k}(x) \not \equiv 0$. Assume in addition that either there exists $x_{0} \in \mathbb{R}$ such that $q_{k}\left(x_{0}\right)<0$ or there exists $x_{0} \in \mathbb{R}$ such that $q_{k}\left(x_{0}\right)=0$ and $q_{k-1}\left(x_{0}\right) \neq 0$. Then $U_{Q}$ does not preserve the set of non-negative polynomials of degree smaller than or equal to $k$.

Proof. The polynomial $p_{t}(x)=(x-t)^{k}$ is non-negative since $k$ is even. Similar to the above one has

$$
r_{Q}(x, t):=\frac{U_{Q}\left(p_{t}\right)(x)}{p_{t}(x)}=q_{0}(x)+k q_{1}(x) \frac{1}{x-t}+\ldots+k ! q_{k}(x) \frac{1}{(x-t)^{k}} .
$$

As before we set $u:=\frac{1}{x-t}$ and consider the function

$$
g(x, u):=q_{0}(x)+k q_{1}(x) u+\ldots+k ! q_{k}(x) u^{k},
$$

which is a polynomial in $u$. If there exists $x_{0}$ such that $q_{k}\left(x_{0}\right)<0$ then $g\left(x_{0}, u\right)$ is a polynomial in $u$ which is negative for sufficiently large values of $u$. If $q_{k}\left(x_{0}\right)=0$ then $g\left(x_{0}, u\right)$ is a polynomial in $u$ of odd degree. In both cases there exists $u_{0}$ such that $g\left(x_{0}, u_{0}\right)<0$. Now for $t_{0}=x_{0}-\frac{1}{u_{0}}$ we get that $r_{Q}\left(x_{0}, t_{0}\right)<0$. Thus $U_{Q}\left(p_{t_{0}}\right)\left(x_{0}\right)<0$ since $p_{t}(x) \geq 0$ for all $(x, t)$. This contradiction accomplishes the proof.

Corollary 4.5. Let $U_{Q}: \mathbb{R}[x] \rightarrow \mathbb{R}[x]$ be a linear differential operator of order $k$ of the form (1) with polynomial coefficients $Q=\left(q_{0}(x), q_{1}(x), \ldots, q_{k}(x)\right), q_{i}(x) \in \mathbb{R}[x]$, $i=1, \ldots, k, q_{k}(x) \not \equiv 0$. Assume that there exists an even integer $i \in\{2,4, \ldots, k\}$ such that either there exists $x_{0} \in \mathbb{R}$ such that $q_{i}\left(x_{0}\right)<0$ or there exists $x_{0} \in \mathbb{R}$ such that $q_{i}\left(x_{0}\right)=0$ and $q_{i-1}\left(x_{0}\right) \neq 0$. Then $U_{Q}$ does not preserve non-negativity in $\mathbb{R}_{l}[x]$ for any $l \geq i$.

Proof. If $l \geq i$ and $U_{Q}$ preserves non-negativity in $\mathbb{R}_{l}[x]$ then $U_{Q}$ preserves nonnegativity in $\mathbb{R}_{i}[x]$. The restriction of $U_{Q}$ to the space $\mathbb{R}_{i}[x]$ is given by

$$
q_{0}(x)+q_{1}(x) \frac{d}{d x}+q_{2}(x) \frac{d^{2}}{d x^{2}}+\ldots+q_{i}(x) \frac{d^{i}}{d x^{i}}
$$

and the result follows from Theorem 4.4.

Remark 4.6. In particular, if $i=2$ in Corollary 4.5 then there is no degree $l$, $l \neq 0$, such that $U_{Q}: \mathbb{R}_{l}[x] \rightarrow \mathbb{R}_{l}[x]$ preserves non-negativity. Thus for a "generic" linear differential operator $U_{Q}: \mathbb{R}[x] \rightarrow \mathbb{R}[x]$ with non-constant coefficients there is no $l$ such that $U_{Q}$ preserves non-negativity in $\mathbb{R}_{l}[x]$. However, the following example shows that there are linear differential operators with non-constant coefficients which preserve positivity on $\mathbb{R}_{k}[x]$ for any even $k$.

Example 4.7. Let $k$ be an even positive integer and consider the linear differential operator of order $k$ given by

$$
U_{k}^{a, b}=1+\left(a x^{k}+b\right) \frac{d^{k}}{d x^{k}}: \mathbb{R}_{k}[x] \rightarrow \mathbb{R}_{k}[x] .
$$

Then $U_{k}^{a, b}$ preserves positivity on $\mathbb{R}_{k}[x]$ if $a \geq 0, b \geq 0$.

Indeed, $U_{k}^{a, b}(p)(x)=p(x)+k ! a_{k}\left(a x^{k}+b\right) \geq p(x)>0$ for any positive polynomial $p(x)=a_{k} x^{k}+\ldots \in \mathbb{R}_{k}[x]$. 


\section{LINEAR ORDINARY DIFFERENTIAL OPERATORS WITH CONSTANT COEFFICIENTS}

In this section we will prove Theorem B. Take a sequence $\alpha=\left(\alpha_{0}, \alpha_{1}, \ldots, \alpha_{k}\right)$ of real numbers. Denote by $U_{\alpha}$ the following linear differential operator of order $k$

$$
U_{\alpha}=\alpha_{0}+\alpha_{1} \frac{d}{d x}+\ldots+\alpha_{k} \frac{d^{k}}{d x^{k}}
$$

with constant coefficients.

By Theorem 4.2 there are no finite order linear differential operators on $\mathbb{R}[x]$ preserving positivity. However, in the case of polynomials of bounded degree, i.e., belonging to the finite-dimensional space $\mathbb{R}_{k}[x]$, there are such linear differential operators, see Example 4.7.

Theorem B follows easily from the next statement of Remak [16] and Hurwitz [5] which for the sake of completeness we present with its proof.

Theorem C. For an even integer $k=2 l$ and a sequence of real numbers $\alpha=$ $\left(\alpha_{0}, \alpha_{1}, \ldots, \alpha_{k}\right)$ consider the linear ordinary differential operator (9) with constant coefficients. Then the operator $U_{\alpha} \neq \equiv 0$ preserves non-negativity in $\mathbb{R}_{k}[x]$ if and only if one of the following two equivalent conditions holds:

(1) for any non-negative polynomial $p(x)=a_{k} x^{k}+\ldots+a_{1} x+a_{0}$ one has that

$$
U_{\alpha}(p)(0)=a_{0} \alpha_{0}+a_{1} \alpha_{1}+\ldots+k ! a_{k} \alpha_{k} \geq 0 ;
$$

(2) the following $(l+1) \times(l+1)$ Hankel matrix

$$
\left(\begin{array}{ccccc}
\alpha_{0} & 1 ! \alpha_{1} & 2 ! \alpha_{2} & \ldots & l ! \alpha_{l} \\
1 ! \alpha_{1} & 2 ! \alpha_{2} & 3 ! \alpha_{3} & \ldots & (l+1) ! \alpha_{l+1} \\
2 ! \alpha_{2} & 3 ! \alpha_{3} & 4 ! \alpha_{4} & \ldots & (l+2) ! \alpha_{l+2} \\
\vdots & \vdots & \vdots & \ddots & \vdots \\
l ! \alpha_{l} & (l+1) ! \alpha_{l+1} & (l+2) ! \alpha_{l+2} & \ldots & (2 l) ! \alpha_{2 l}
\end{array}\right)
$$

represents a positive semi-definite quadratic form. Recall again that unlike positive definite and positive semidefinite sequences we assume by definition that a positive definite quadratic form is also positive semi-definite.

We start with the following observation.

Lemma 5.1. The operator $U_{\alpha}: \mathbb{R}_{k}[x] \rightarrow \mathbb{R}_{k}[x]$ of the form (9) commutes with shifts of the independent variable $x$. In other words, for any polynomial $p(x) \in \mathbb{R}_{k}[x]$ set $q(x)=U_{\alpha}(p)(x)$. Then for any $x_{0} \in \mathbb{R}$ we have that $q\left(x-x_{0}\right)=U_{\alpha}(p)\left(x-x_{0}\right)$.

Proof. Take any $p(x)=a_{l} x^{l}+\ldots+a_{0}, l \leq k$. Then for any positive integer $i$ we have that $p^{(i)}(x)=a_{l} \frac{l !}{(l-i) !} x^{l-i}+\ldots+a_{i} i$. Thus $\left(p^{(i)}\right)\left(x-x_{0}\right)=\left(p\left(x-x_{0}\right)\right)^{(i)}$. Since the coefficients of $U_{\alpha}$ are constant, the result follows.

Proof of Theorem C. The equivalence between the conditions (1) and (2) in the formulation of Theorem $\mathrm{C}$ is exactly the same fact as the equivalence between (2) and (3) in Theorem 3.4 for $\lambda_{0}=\alpha_{0}, \lambda_{i}=i ! \alpha_{i}, i=1, \ldots, k$ which is valid both in the finite-dimensional and infinite-dimensional cases. What we need is to show that the assumption that $U_{\alpha}$ is a non-negativity-preserver in $\mathbb{R}_{k}[x]$ is equivalent to condition (1). Indeed, if $U_{\alpha}$ preserves non-negativity in $\mathbb{R}_{k}[x]$, then $a_{0} \alpha_{0}+a_{1} \alpha_{1}+\ldots+$ $k ! a_{k} \alpha_{k}=U_{\alpha}(p)(0) \geq 0$ for any non-negative polynomial $p(x) \in \mathbb{R}_{k}[x]$. Assume now that for any non-negative polynomial $p(x)$ one has that $a_{0} \alpha_{0}+a_{1} \alpha_{1}+\ldots+k ! a_{k} \alpha_{k} \geq$ 0 . Set $q(x):=U_{Q}(p)(x)$. By assumption we have that $q(0) \geq 0$ and we want to show that $q(x)$ is non-negative. For any $x_{0} \in \mathbb{R}$ consider $g_{x_{0}}(x):=q\left(x+x_{0}\right)$. By Lemma 5.1 we have that $g_{x_{0}}(x)=U_{\alpha}(p)\left(x+x_{0}\right)$, but $f(x):=p\left(x+x_{0}\right)$ is a non-negative polynomial. Thus by condition (1) one has $U_{\alpha}(f)(0) \geq 0$, i.e.,

$$
q\left(x_{0}\right)=g_{x_{0}}(0)=U_{\alpha}(f)(0) \geq 0
$$


for any $x_{0} \in \mathbb{R}$.

Remark 5.2. Theorem $\mathrm{C}$ provides the classification of linear differential operators with constant coefficients of an even order $k$ which preserve non-negativity in $\mathbb{R}_{k}[x]$. On the other hand, by Theorems A and 4.2 there are no linear differential operators with constant coefficients of even order $k$ that preserve positivity in $\mathbb{R}_{2 k}[x]$. Below we bridge this gap between $k$ and $2 k$ for operators with constant coefficients by showing that there are no such operators of order $k$ that preserve positivity (or non-negativity, or ellipticity) in $\mathbb{R}_{l}[x]$ for any $l>k$.

Proposition 5.3. Let $k$ be a positive integer and let $\alpha=\left(\alpha_{0}, \alpha_{1}, \ldots, \alpha_{k}\right)$ be $a$ sequence of real numbers. Consider the operator $U_{\alpha}$ of the form (9). Then for any $l>k$ the operator $U_{\alpha}: \mathbb{R}_{l}[x] \rightarrow \mathbb{R}_{l}[x]$ does not preserve positivity.

Proof. Wlog we can assume that $l$ is even. We can also assume $\alpha_{0}>0$ and at least one more entry $\alpha_{j}$ in the sequence $\left(\alpha_{0}, \alpha_{1}, \ldots, \alpha_{k}\right)$ is non-vanishing. (The cases when either $\alpha_{0} \leq 0$ or only $\alpha_{0}$ is non-vanishing are trivial.) Take any (not necessarily positive!) polynomial $p(x)=a_{k} x^{k}+\ldots+a_{1} x+a_{0}$ of degree at most $k$ such that $a_{0}>0$ and $U_{\alpha}(p)(0)=a_{0} \alpha_{0}+a_{1} \alpha_{1}+\ldots+k ! a_{k} \alpha_{k}<0$. Since both $\alpha_{0}$ and $\alpha_{j}$ are non-vanishing such a $p(x)$ always exists. Consider now $P(x)=M x^{l}+p(x)$ where $M$ is a large positive constant. By our assumptions one can always choose such a large $M$ that $P(x)$ becomes positive. At the same time $U_{\alpha}(P)(0)=U_{\alpha}(p)(0)<0$. The latter contradicts to the condition (1) of Theorem $\mathrm{C}$ implying that $U_{\alpha}$ does not preserve positivity in $\mathbb{R}_{l}[x]$.

Let us finally deduce Theorem B from Theorem C and Theorem 3.4.

Proof. Observe that for any positive even integer $k$ the action of the operator $U_{\alpha}$ of infinite order of the form (3) on the space $\mathbb{R}_{k}[x]$ coincides with the action of its truncation (9). Moreover, by Lemma 2.1 any such $U_{\alpha} \not \equiv 0$ of the form (3) preserves positivity if and only if it preserves non-negativity. Condition (1) of Theorem $\mathrm{C}$ claims that the sequence $\left\{k ! \alpha_{k}\right\}$ is positive. Therefore by condition (4) of Theorem 3.4 there exists a positive measure $\mu_{\alpha}$ with all finite moments such that for any $k=0,1,2, \ldots$ one has $k ! \alpha_{k}=\int_{-\infty}^{\infty} t^{k} d \mu(t)$, i.e. $k ! \alpha_{k}$ is the $k$-th moment of $\mu_{\alpha}$. Let us show that for any polynomial $p(x)$ one has $U_{\alpha}(p)(x)=$ $p(x) \star \mu_{\alpha}=\int_{-\infty}^{\infty} p(t) d \mu_{\alpha}(t-x)$. Let us first settle the statement at the origin. If $p(x)=a_{k} x^{k}+\ldots+a_{1} x+a_{0}$ then one immediately checks that $U_{\alpha}(p)(0)=$ $\int_{-\infty}^{\infty} p(t) d \mu_{\alpha}(t)=\alpha_{0} a_{0}+\alpha_{1} a_{1}+2 ! \alpha_{2} a_{2}+\ldots+k ! \alpha_{k} a_{k}$. Further, since $U_{\alpha}$ is translation invariant one gets for any real $x_{0}$ that $U_{\alpha}(p)\left(x_{0}\right)=\alpha_{0} b_{0}+\alpha_{1} b_{1}+2 ! \alpha_{2} b_{2}+\ldots+k ! \alpha_{k} b_{k}$ where $p(x)=b_{0}+b_{1}\left(x-x_{0}\right)+\ldots+b_{k}\left(x-x_{0}\right)^{k}$. At the same time one has

$\int_{-\infty}^{\infty} p(t) d \mu_{\alpha}\left(t-x_{0}\right)=\int_{-\infty}^{\infty} p\left(\bar{t}+x_{0}\right) d \mu_{\alpha}(\bar{t})=\alpha_{0} b_{0}+\alpha_{1} b_{1}+2 ! \alpha_{2} b_{2}+\ldots+k ! \alpha_{k} b_{k}$,

since the coefficients of expansion of the polynomial $p\left(\bar{t}+x_{0}\right)$ w.r.t. the variable $\bar{t}$ are exactly $b_{0}, b_{1}, \ldots, b_{k}$.

Similar considerations can be found in Ch. 7 of [7].

Remark 5.4. Let us explain why there exist positivity-preservers which are differential operators with constant coefficients and which are different from the inverses of hyperbolicity-preservers given by (finite and infinite order) linear differential operators with constant coefficients. Namely, Theorem 2 of [1] claims that the inverse of such a hyperbolicity-preserver is either a shift operator $e^{a \frac{d}{d x}}$ for some real $a$ or the convolution (over $\mathbb{R}$ ) with a Polya frequency density function. (About Polya frequency functions consult e.g. Ch. 7 of [7].) It is well-known that any Polya 
frequency density function is unimodular. Therefore the convolution with any nonunimodular positive and quickly decreasing kernel gives a required example.

\section{REFERENCES}

[1] J. Carnicer, J. Peña, A. Pinkus, On some zero-increasing operators, Acta Math.Hung. 94 (3), (2002), 173-190.

[2] T. Craven, G. Csordas, Problems and theorems in the theory of multiplier sequences, Serdica Math. J. 22 (1996), 515-524.

[3] T. Craven, G. Csordas, Complex zero decreasing sequences, Methods Appl. Anal. 2 (1995), 420-441.

[4] R. Ehrenborg, The Hankel determinant of exponential polynomials. Amer. Math. Monthly 107 (2000), no. 6, 557-560.

[5] A. Hurwitz, Über definite Polynome, Math. Ann. 73 (1913), 173-176.

[6] L. Iliev, Laguerre entire functions. 2nd ed., Publ. House of the Bulgarian Acad. Sci., Sofia, 1987, $188 \mathrm{pp}$

[7] S. Karlin, Total positivity. Vol. I. Stanford University Press, Stanford, Calif 1968 xii+576 pp.

[8] M. D. Kostova, Über die $\lambda$-Folgen (German) [On $\lambda$-sequences], C. R. Acad. Bulgare Sci. 36 (1983), 23-25.

[9] J. S. Lomont, J. Brillhart, Elliptic polynomials. Chapman \& Hall/CRC, Boca Raton, FL, 2001, xxiv+289 pp.

[10] T. S. Motzkin, Algebraic inequalities, in "Proc. Sympos. Wright-Patterson Air Force Base," Ohio, 1965, pp. 199-203, Academic Press, New York.

[11] I. P. Natanson, Konstruktive Funktionentheorie. (German) Akademie-Verlag, Berlin, 1955. xiv +515 pp.

[12] F. B. Pakovich, Elliptic polynomials (Russian), Uspekhi Mat. Nauk. 50 (1995), 203-204; English translation in Russian Math. Surv. 50 (1995), 1292-1294.

[13] A. Prestel, C. N. Delzell, Positive polynomials. From Hilbert's 17th problem to real algebra. Springer Monographs in Mathematics, Springer-Verlag, Berlin, 2001, viii+267 pp.

[14] G. Pólya, I. Schur, Über zwei Arten von Faktorenfolgen in der Theorie der algebraischen Gleichungen, J. Reine Angew. Math. 144 (1914), 89-113.

[15] G. Pólya, G. Szegö, Problems and theorems in analysis. II. Theory of functions, zeros, polynomials, determinants, number theory, geometry. Translated from the German by C. E. Billigheimer. Reprint of the 1976 English translation. Classics in Mathematics. SpringerVerlag, Berlin, 1998. xii+392 pp.

[16] R. Remak, Bemerkung zu Herrn Stridsbergs Beweis des Waringschen Theorems, Math. Ann. 72 (1912), 153-156.

[17] D. V. Widder, The Laplace Transform. Princeton Math. Series Vol 6, Princeton Univ. Press, Princeton, NJ, 1941, x+406 pp.

Faculty of Algebra, Department of Mathematics and Mechanics, Moscow State UniVERSity, 119991, GSP-1, Moscow, Russia

E-mail address: guterman@list.ru

Department of Mathematics, Stockholm University, SE-106 91 Stockholm, Sweden

E-mail address: shapiro@math.su.se 